Open Systems \& Information Dynamics

Vol. 26, No. 4 (2019) 1999001 (2 pages)

DOI:S1230161219990014

(C) World Scientific Publishing Company

$\| \beta$ World Scientific

\title{
Index of Vol. 26 (2019)
}

Accardi L. And F. Guerrero-Poblete, Quantum Markov Semigroups of Low Density Limit: the Generic Case

Amini N. H. And J. E. Gough, The Estimation Lie Algebra Associated with Quantum Filters

1950004

AnzÀ F., A. Messina, And B. Militello, Resonant Transitions Due to Changing Boundaries

Asorey M., P. Facchi, And G. Marmo, Topological Order, Mixed States and Open Systems

Aspuru-Guzik A., see Rodríguez-Rosario C. A., T. Frauenheim, And A. AspuruGUZIK

1950006

1950012

1950022

Avron J. E., see Tsabary A., O. Kenneth, and J. E. Avron 1950008

Benatti F., Hadamard Completely Positive Semigroups

1950020

Chruściński D. And A. Kossakowski, Divisibility of Dynamical Maps with Time Independent Invariant State

1950019

di Cosmo F., A. Ibort, And G. Marmo, Groupoids and Coherent States

1950017

Dirr G., see vom Ende F., G. Dirr, M. Keyl, and T. Schulte-Herbrüggen

1950014

Dunningham J., see Palge V., J. Dunningham, S. Groote, and H. Liivat

1950001

vom Ende F., G. Dirr, M. Keyl, and T. Schulte-HerbrügGen, Reachability in Infinite-Dimensional Unital Open Quantum Systems with Switchable GKS-Lindblad Generators

1950014

Facchi P., see Asorey M., P. FACChi, And G. Marmo

1950012

Fagnola F. And C. M. Mora, Basic Properties of a Mean Field Laser Equation

1950015

Frauenheim T., see Rodríguez-Rosario C. A., T. Frauenheim, and A. AspuruGUZIK

Gough J. E., see Amini N. H. And J. E. Gough

1950004

Groote S., see Palge V., J. Dunningham, S. Groote, and H. Limvat

1950001

Guerrero-Poblete F., see Accardi L. and F. Guerrero-Poblete

1950021

Ibort A., see di Cosmo F., A. Ibort, And G. Marmo

1950017

Kamizawa T., Reducibility Criteria and a Construction Method for the Analysis of Open Quantum Systems

1950010

Kenneth O., see Tsabary A., O. Kenneth, and J. E. Avron

1950008

Keyl M., see vom Ende F., G. Dirr, M. Keyl, and T. Schulte-Herbrüggen

1950014

Kossakowski A., see Chruściński D. And A. Kossakowski

1950019 
Author Index

Liivat H., see Palge V., J. Dunningham, S. Groote, and H. Lifvat

1950001

Lorenzo S., M. Paternostro and G. M. Palma, Reading a Qubit Quantum State with a Quantum Meter: Time Unfolding of Quantum Darwinism and Quantum Information Flux

MaćKowiak J., Bose Liquid Mean-Field Theory with Application to HeI

MAN'Ko M. A. AND V.I. MAN'KO, Interference of Quantum States and Superposition Principle in the Probability Representation of Quantum Mechanics

1950016

Ma'NKO V. I., see MAN'KO M. A. AND V. I. MAN'KO

1950016

Marmo G. and S. Pascazio, The Legacy of George Sudarshan

1950011

Marmo G., see Asorey M., P. Facchi, and G. Marmo

1950012

Marmo G., see di Cosmo F., A. Ibort, and G. Marmo

1950017

Messina A., see Anzà F., A. Messina, and B. Militello

1950006

Militello B.., see Anzì F., A. Messina, And B. Militello 1950006

Modi K., George Sudarshan and Quantum Dynamics 1950013

Mora C. M., see Fagnola F. And C. M. Mora

1950015

Ohmura K. and N. Watanabe, Quantum Dynamical Mutual Entropy Based on AOW

1950009

Palge V., J. Dunningham, S. Groote, and H. Liivat, Maps Generated by Entangled Momenta: Exploring Spin Entanglement in Relativity

1950001

Palma G. M., see Lorenzo S., M. Paternostro and G. M. Palma 1950023

Pascazio S., see Marmo G. and S. Pascazio

1950011

Paternostro M., see Lorenzo S., M. Paternostro and G. M. Palma

1950023

Pathak V. AND A. Shaji, Non-Markovian Open Dynamics from Collision Models 1950018

Rebolledo R., Contiguity of States and Super Wave Operators

1950002

Rodríguez-Rosario C. A., T. Frauenheim, and A. Aspuru-Guzik, Quantum Coherences as a Thermodynamic Potential

1950022

Schulte-Herbrüggen T., see vom Ende F., G. Dirr, M. Keyl, and T. SchulteHERBRÜGGEN

1950014

Shaji A., see Pathak V. AND A. ShajI

1950018

TadeJ W., Defect and Equivalence of Unitary Matrices. The Fourier Case. Part II

1950003

Tsabary A., O. Kenneth, and J. E. Avron, Anomalous Decay and Decoherence in Atomic Gases

1950008

Watanabe N., see Ohmura K. and N. Watanabe

1950009

Wichert A., Principles of Quantum-like Evolution

1950007 